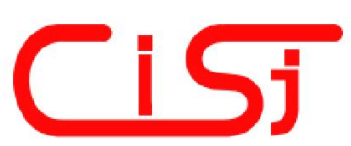

\title{
PRECISE SYNCHRONISATION IN INDUSTRIAL SYSTEM USING IEEE 1588 PRECISE TIME PROTOCOL
}

\author{
Vladimir Haasz ${ }^{1)}$, Jaroslav Roztocil ${ }^{2)}$, Jan Breuer ${ }^{2)}$, Vojtěch Vigner ${ }^{2)}$ \\ ${ }^{1)}$ Czech Technical University in Prague, University Centre for Energy Efficient Buildings, \\ Třinecká 1024, 27343 Buštěhrad, Czech Republic \\ ${ }^{2)}$ Czech Technical University in Prague, Faculty for Electrical Engineering, \\ Technická 2, 16627 Prague 6, Czech Republic \\ \{haasz, roztocil, jan.breuer, vignevoj\}@fel.cvut.cz, http://measure.feld.cvut.cz/lide
}

\begin{abstract}
Nowadays there is a frequent demand to increase the quality of synchronization in industrial distributed systems. Node synchronization can be implemented in the Ethernet network using time protocols, e.g. IEEE 1588 Precise Time Protocol (PTP). However, active network components like switches and routers have negative influence on achieved accuracy of synchronization because they affect a packet delay variation in the network. To suppress this effect, special (but expensive) industrial switches, with a packet propagation delay correction, can be used. Another way using either low cost GPS receivers for synchronization of local clocks or a special timing for data transfer is described in the paper. It will allow a cheaper solution that preserves high quality of synchronization. Besides, a device for packet delay measurement (PTP tester) was designed and developed to be possible to evaluate the proposed methods. Copyright (C) Research Institute for Intelligent Computer Systems, 2015. All rights reserved.
\end{abstract}

Keywords: industrial distributed systems, synchronization, Precise Time Protocol, GPS, packet propagation delay.

\section{INTRODUCTION}

Real time operation is a very important aspect of industrial distributed systems. A special software and network components are applied in this case. However, the correct function of such systems depends also on proper time synchronization of all single subsystems. Time synchronization can be accomplished by several different approaches. Each of these approaches is suitable for specific applications. Synchronization of two or more clocks can be done using direct clock distribution in industrial buses or by radio transmitted synchronization signal in larger systems (e.g. GPS, GLONASS or Galileo [1]).

In the first case, node synchronization can be implemented directly in the Ethernet network using time protocols, e.g. IEEE 1588 Precise Time Protocol (PTP) [2]. In this case, the accuracy of synchronization can be negatively influenced by active network components like switches and routers, which delay packet transmission. Unfortunately this delay changes depending on a number of factors.

In the second case, the GPS is utilized to provide synchronization of systems without a special synchronization line. This solution is suitable for time synchronization in large industrial systems. Furthermore, such a modular solution can be implemented without significant rebuilding of existing systems. It can be achieved by replacing of an existent master clock device with a new one synchronized by a GPS receiver.

The time uncertainty better than $10 \mu$ s can be achieved by joint of the both technologies mentioned above. This solution seems to be very perspective, but a real application is not simple. To achieve such high precision of synchronization requires a very sophisticated design. It is necessary to know a number of parameters of used network components, especially switches. The problem is also in a relatively limited offer of suitable commercially produced network components (e.g. control components and actuators with implemented PTP) for reasonable price.

The quality of synchronization in the Ethernet network depends on the packet delay variation caused by an underlying network, on the precision of time stamping in the terminal nodes, and stability of the time base in these nodes. A packet delay variation can be compensated by special network elements. Precise time stamping can be performed on the Ethernet physical layer (PHY). A stable time base can be ensured by either a temperature 
compensated crystal oscillator (TCXO), an ovencontrolled crystal oscillator (OCXO) or using an oscillator synchronized by a GPS receiver. The features of net components and GPS receivers concerning this application should be evaluated before the finalization of its design.

\section{EVALUATION OF PARAMETERS OF THE ETHERNET NETWORK CONCERNING PRECISE SYNCHRONIZATION}

Implementation of PTP in the Ethernet network is not trivial. Active network components like switches and routers influence the accuracy of synchronization because they affect the packet delay in the network. Unfortunately this delay is not constant and moreover there is a difference between the one transfer direction and the opposite one.

The existing ways of testing using low-cost instrumentation appreciate the secondary effects only, because the properties of network concerning time parameters are not measured directly, but only a possibility to synchronize slave devices to the master device is evaluated. However, this way is not suitable for evaluation of a synchronization accuracy, because it is influenced by many secondary actions, e.g. with control algorithm of slave device etc. A direct measurement of a packet delay over an underlying network requires a special and expensive measuring device [3, 4]. Therefore a low cost PTP tester was designed and developed. It enables a direct measurement of time parameters of the network with a direct monitoring of single packets and time of their transmission. The direct measurement ensures a more precise evaluation of network properties and determination of further parameters, which cannot be determine only by monitoring of synchronization quality of the slave device.

The basic parameter, which is measured, is the delay of packet transmission between two nodes. The following parameters (metrics) of transmission path are calculated from these parameters:

\section{Packet Delay Variation (PDV)}

PDV is the difference of the delay of the actual packet and the delay of previous packet in time dependence (possibly the first or the second derivation of these values). The value of PDV should be minimum for precise synchronization. If the delay of packets is constant, then it is possible to measure and to compensate it.

\section{Asymmetry}

Asymmetry is a different delay in the same time, but in both opposite transfer directions. It can be measured only if the testing device acquires the time information of the both nodes using a separate synchronization channel without asymmetry (or if it is known and constant).

Other advanced metrics are e.g. Maximum Time Interval Error (MTIE) and Time Deviation.

\subsection{THE PACKET DELAY MEASURING DEVICE (PTP TESTER)}

The main component of the PTP tester is an integrated circuit IEEE 1588 Precision Time Protocol Transceiver Physical Layer (PHY) DP83630 by Texas Instruments (see [5]). It allows to time stamp ingoing and outgoing packets directly in the PHY interface. Thanks to this function, it is possible to reach a high resolution of time stamps (in this case $8 \mathrm{~ns}$ ). In order to function properly, the device should have a synchronous time scale. The basic connection is shown in Fig. 1.

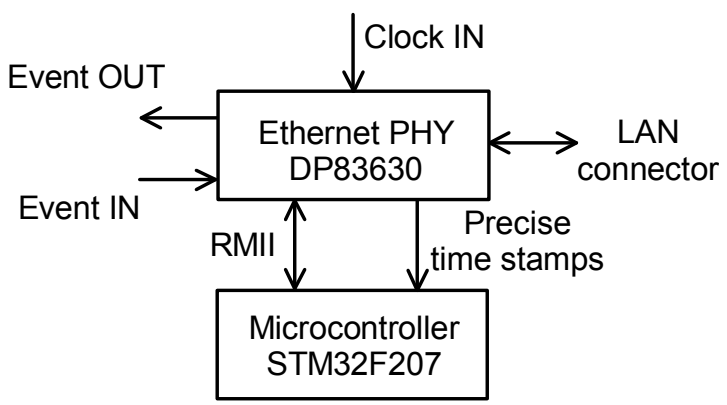

Fig. 1 - The basic connection of the PTP tester.

For local measurement, a synchronous time scale can be simply achieved using the same clock source for the both nodes in the device (see Fig. 2).

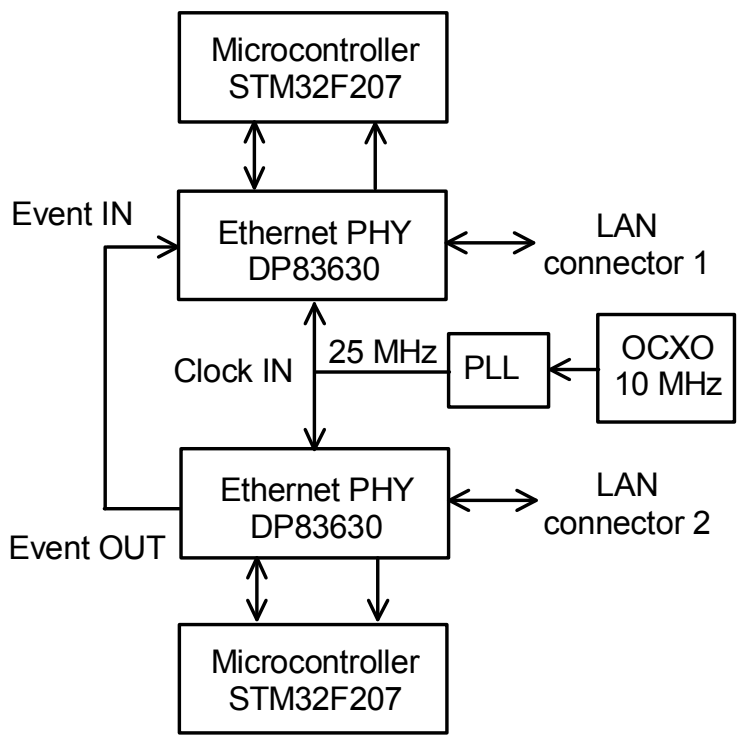

Fig. 2 - Connection of the PTP tester - modification for local measurement.

To measure an interval precisely, the system clock should have sufficient stability (see [6]). Such 
stability is probed by e.g. the $10 \mathrm{MHz}$ OCXO MTI-210 (see [7]). The nodes are fully synchronous in this case, which enables to measure both the delay of the network connection and also its asymmetry.

A different situation comes in the case of large distributed systems. In this case it is not possible to use the same clock source for both nodes in the device. The testing device has to be divided in two parts (2 same separate modules), where each one has its individual clock controlled by separate GPS receivers (see Fig. 3). However, it is necessary to combine a GPS receiver with an OCXO, which has better short-term stability, to maintain the precise time scale. The connection of one module of the PTP tester modified for testing of large distributed systems is presented in Fig. 4.

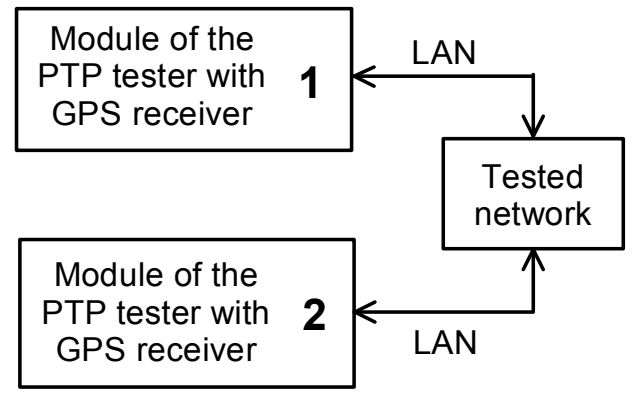

Fig. 3 - Modification of the PTP tester designed for testing of large distributed systems.

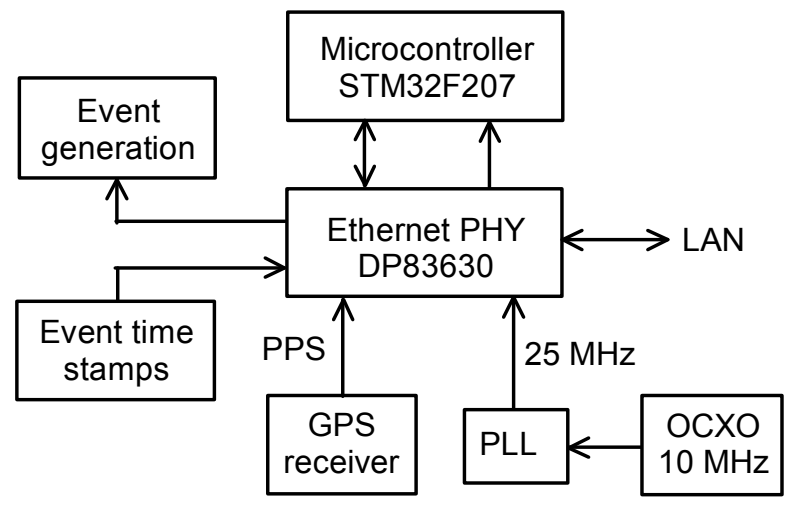

Fig. 4 - One module of the PTP tester - modification for distributed measuring system.

\subsection{EVALUATION OF THE PTP TESTER}

The offset of the PTP tester caused e.g. by PHY and LAN connectors has to be determined before its application. Two ways of measurement were applied to estimate it.

In the simplest way it is possible to use UTP cables with different lengths as a delay line. At first, a transport delay of the cables was measured by the SR620 Stanford Research Universal Time Interval Counter and this value was considered as the reference value (see Table 1). The delay values measured by the PTP tester were subtracted from the values measured by a counter. This difference corresponds to the basic offset of the PTP tester. At least one thousand of measurements were performed using the same cable. A final mean value of offset of $20 \pm 1 \mathrm{~ns}$ can be afterwards used for correction of all measured data. Differences among single measurements (between reference and measured values) correspond to the resolution of time stamps $(8 \mathrm{~ns})$. This offset was numerically corrected during all following tests.

Table 1. Measurement using UTP cables as a delay line.

\begin{tabular}{|c|c|c|c|c|}
\hline Length (m) & 1 & 5 & 9.5 & 50 \\
\hline $\begin{array}{c}\text { Delay measured by } \\
\text { counter (ns) }\end{array}$ & 10 & 27 & 49 & 241 \\
\hline $\begin{array}{c}\text { Delay measured by } \\
\text { the PTP tester (ns) }\end{array}$ & 216 & 232 & 256 & 448 \\
\hline $\begin{array}{c}\text { Offset of the PTP } \\
\text { tester (ns) }\end{array}$ & 206 & 205 & 207 & 207 \\
\hline
\end{tabular}

Nevertheless, the maximal delay which can be realized using a UTP cable connected between individual nodes is limited. For longer packet delays it is necessary to use a more sophisticated device, a packet delay simulator, e.g., the Calnex Paragon X device developed by the Calnex Solutions Ltd. is suitable. It can simulate not only a constant packet delay on the Ethernet network, but also dynamic changes of this delay. The following measurements were done in the Calnex laboratory. The PTP tester was connected with the Calnex Paragon $\mathrm{X}$ using the 100BASE-TX Ethernet and the separate $10 \mathrm{MHz}$ clock signal for synchronization (see Fig. 5).

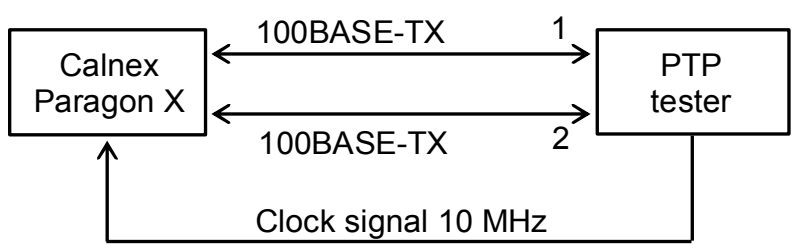

Fig. 5 - Measurement setup.

Several predefined delays $T_{\mathrm{s}}$ were set in the Calnex Paragon X and a delay of the transfer path $T_{\mathrm{a}}$ was measured by the PTP tester. The values of differences $T_{\mathrm{d}}$ between predefined and measured delays were calculated using as $T_{\mathrm{d}}=T_{\mathrm{a}}-T_{\mathrm{s}}-T_{\mathrm{p}}-2 T_{\mathrm{c}}$; where $T_{\mathrm{a}}$ is a measured delay, $T_{\mathrm{s}}$ is a predefined delay, $T_{\mathrm{p}}$ is a fix pass through delay of the Calnex Paragon-X, and $T_{\mathrm{c}}$ is the delay of the cable. The mean value of $T_{\mathrm{d}}$ was determined about $38 \mathrm{~ns}$ for set delays $T_{\mathrm{s}}$ from 0.5 to $10 \mathrm{~ms}$ (see [8] for detail).

The path delay was measured in both directions. A small asymmetry of about 56 ns was found out. The same measurement setup was used with a 
variable delay as well. The generated delay was changed according a saw shape with the amplitude of $1 \mu \mathrm{s}$ or $10 \mu \mathrm{s}$ and the basic delay of $50 \mu \mathrm{s}$. The measured values correspond to the results of previous measurement with the constant set delay.

\subsection{TESTING OF THE DEVICE IN A REAL NETWORK}

Measurement of a packet delay on LAN switches was executed for testing of the developed device in real applications. A cheap common switch for home applications and an industrial switch with and without a packet propagation delay information were used. The evaluated switches were connected to the both LAN connectors to the PTP tester in modification for local measurement (see Fig. 2). The delay about $9.8 \mu$ s with variations about $250 \mathrm{~ns}$ and an asymmetry about 100 ns was measured using a cheap common switch designed for home applications. In the case of an industrial switch with a packet propagation delay information, which is gained from correction field of an IEEE 1588 message, the mean value of delay after correction was determined of $12 \mathrm{~ns}$, the standard deviation of $7 \mathrm{~ns}$ and no asymmetry was found out.

The executed tests proved applicability of the developed PTP tester for packed delay measurement in LAN. The measured data can be used for a decision if the evaluated LAN can be used for application of PTP protocol and to estimate pertinently what accuracy of synchronization can be achieved.

\section{APPLICATION OF GPS TIME SIGNAL FOR TIME SCALE SYNCHRONISATION IN REMOTE NODES}

In the case that synchronization of two or more clocks cannot be achieved by a direct clock distribution using an industrial bus (e.g. IEEE 1588), a radio transmitted synchronization signal (e.g. GPS) can be used. Global Positioning System (GPS) enables worldwide continuous precise time transfer. Special timing GPS receivers are primarily intended for the generation of a precise time scale synchronized with the GPS Time. They generate 1 PPS signal (frequency of $1 \mathrm{~Hz}$ ), which physically represents seconds of the GPS time scale synchronized with the UTC (USNO). This signal is commonly used as the main synchronization signal of local time bases. Another useful feature of some of these receivers is the direct time stamping of external events. This can be utilized for measurement and calibration of external sources of the 1 PPS signal.

The 1 PPS signal from a timing GPS receiver provides a high long-term stability of time scale.
However, its short-term stability is in the most of cases inadequate for precise measurement. For that reason, precise GPS disciplined oscillators are sometimes being used. This combination benefits from good short-term stability of the oscillator signal and great long-term stability of the GPS signal.

Timing GPS receivers automatically apply corrections to the signal reception and processing (processing of $\mathrm{C} / \mathrm{A}$ code from several satellites, usage of synchronization algorithm for connecting internal oscillator, etc.). It outputs a 1 PPS time signal and information about time of the rising edge of the pulse is included in the respective communication protocol (e.g. NMEA). Still, it is necessary to eliminate some of the common sources of errors, particularly: an antenna cable delay, an antenna position fix error, a quality of output PPS signal, a distribution units delay and an output cable delay.

\subsection{SYNCHRONIZATION OF MASTER CLOCK USING GPS RECEIVER}

The master clock, which used synchronization by a GPS receiver, was developed for time stamping in remote nodes. It can be applied for implementation of PTP protocol IEEE 1588. Its connection corresponds to the connection of one module of the PTP tester in modification for testing of large distributed systems (see Fig. 3 above). The excellent quality of time stamping is achieved using precise OCXO oscillator (MTI 210 from MTI Miliren) and a GPS receiver (LEA-6T uBlox), which ensure the long term stable time scale for the whole subsystem (see more in [9]). This solution enables a full independence of a superior system.

\subsection{TESTING OF THE DEVICE IN A REAL NETWORK}

Measurement of a packet delay in a larger network was executed for testing of the PTP tester (in the modification for testing of large distributed measuring systems) in real application. The LAN in the campus of the Czech Technical University in Prague was used for this purpose. The packet delay, the packet delay variation and the asymmetry were measured. The results are presented in Fig. 6 and Fig. 7.

\section{DISTRIBUTED MEASURING SYSTEM WITH IEEE 1588 PROTOCOL}

To test an implementation of the methods of synchronization mentioned above, the simple distributed DAQ system was designed and developed (see Fig. 8). The single nodes distributed measuring channels, which can be 
connected to any sensors with voltage output - are shown also in Fig. 8.

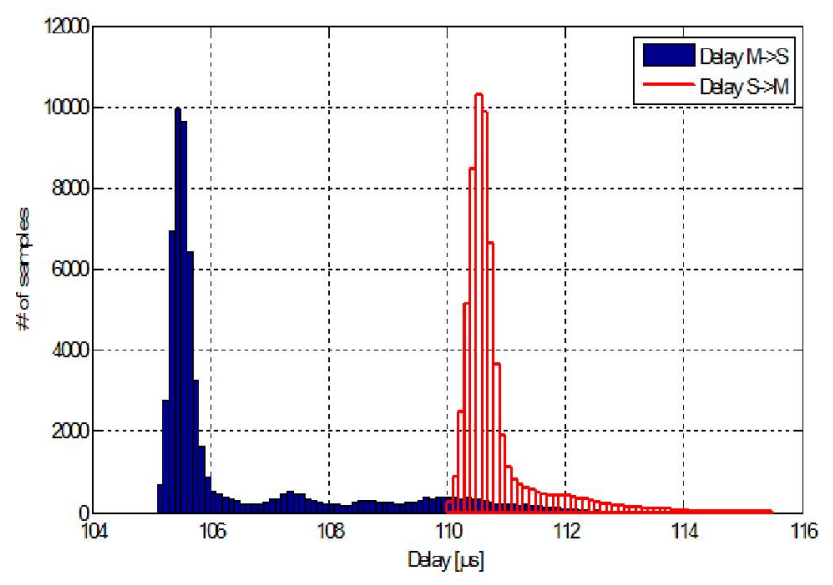

Fig. 6 - Histogram of packed delay in the both directions.

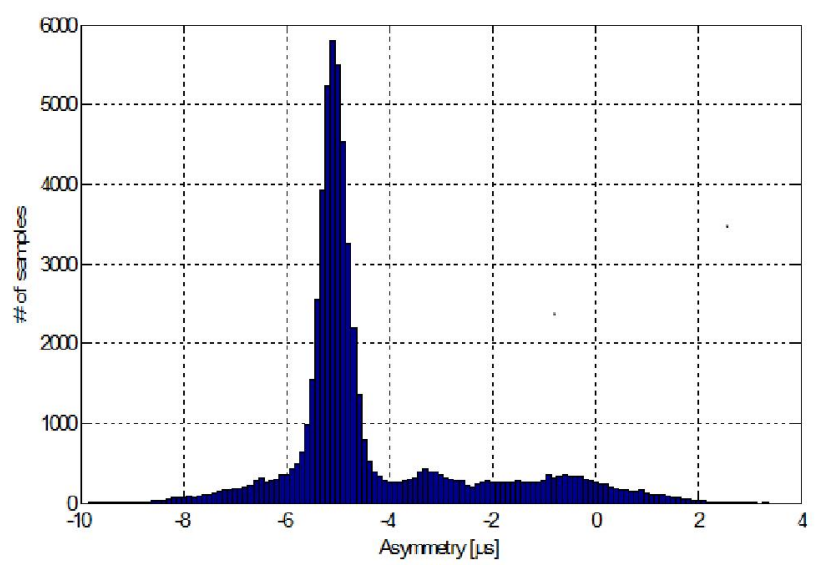

Fig. 7 - Histogram of asymmetry.

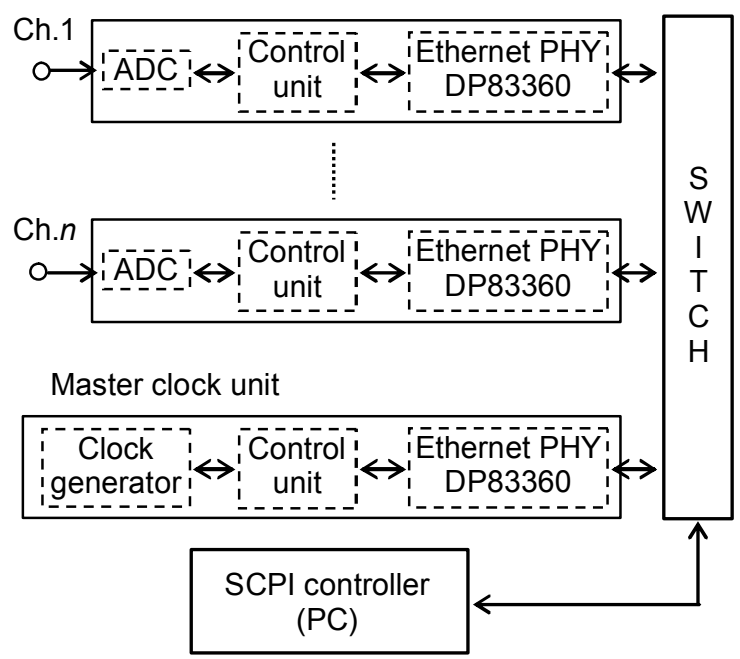

Fig. 8 - Simple distributed DAQ system with IEEE 1588.

All possible basic functions (AD conversion, internal control and Ethernet communication) are implemented in microcontroller. The integrated circuit IEEE 1588 Precision Time Protocol Transceiver Physical Layer (PHY) DP83630 is added to ensure synchronization. The single nodes, the control unit and the master clock are connected together using common Ethernet switch. Each node includes a local clock generator, but it is not possible to use it for precise synchronization due to its bad frequency stability. Therefore the master clock is applied for synchronization of all local clocks using synchronization messages according to IEEE 1588.

This system enables to apply single nodes in distance corresponding to the used Ethernet standard (about $100 \mathrm{~m}$ ). For a longer distance an optical link can be used according to Fig. 9. However, the synchronization accuracy using the PTP protocol is decreased in this case (about several tens of microseconds). It is caused by the low-cost Ethernet optical link converters. If a better quality is demanded, this remote subsystem has to include a separate master clock, which is synchronized using GPS.

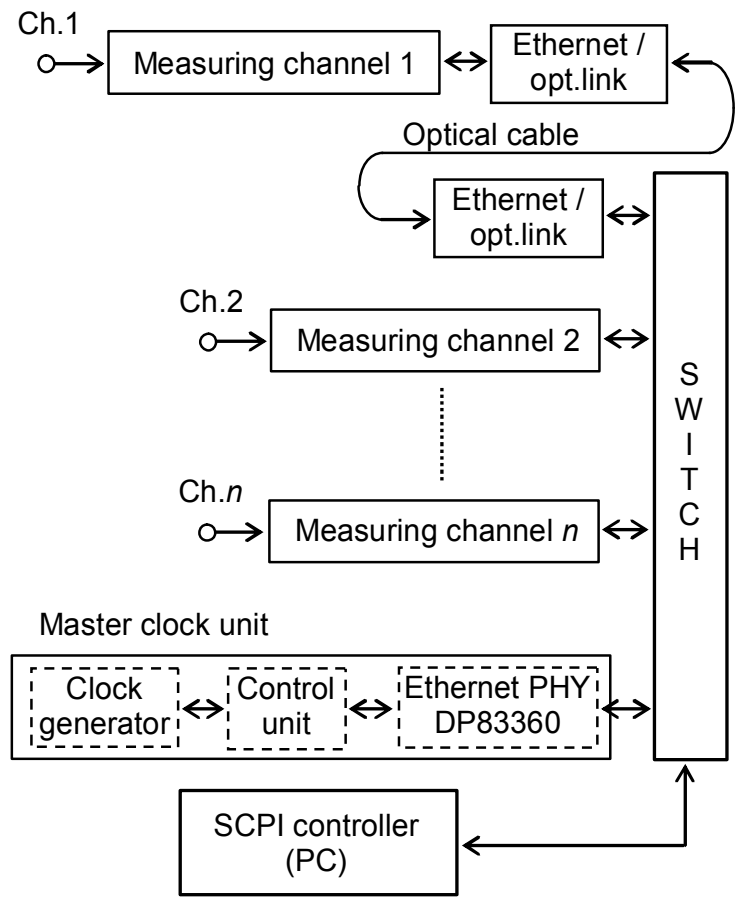

Fig. 9 - Simple distributed DAQ system with a remote subsystem.

Quality of synchronization depends (besides other things) also on properties of used switch(es), especially if the delay of synchronization messages transmitted between switch modules is not constant and not the same in both directions. To evaluate possibility to use a cheap common switch also in this case, the low cost switch TL-SF1008P (TP-LINK) was applied.

Unfortunately the executed experiment confirms the supposed problem. The measured results shown 
that the used switch changes its transfer delay in dependence on its loading. For a small traffic the delay fluctuation was in microseconds range, but it achieved up to several tens of microseconds for the full loading. Nevertheless, a convenient solution was found. A modified way for transmission of the synchronization messages was designed. It is based on a time multiplex, which separates the time window for the synchronization messages from the time windows for measured data transmission.

This solution is presented in Fig. 10. The data flow includes, except of measured data transferred from single modules (data1, data2, ...), also synchronization packets of the PTP protocol $(\mathrm{SC}=$ Sync,$\quad \mathrm{FU}=$ Follow $\mathrm{Up}, \quad \mathrm{DRQ}=$ Delay Request, DRS $=$ Delay Response). For good time synchronization it is necessary to enable "free" flow of these synchronization packets without collisions with measured data. The designed time multiplex takes it into account and no module can start a data transfer before the exchange of the synchronization messages in the actual synchronization period of the PTP protocol (in this application one time per second).

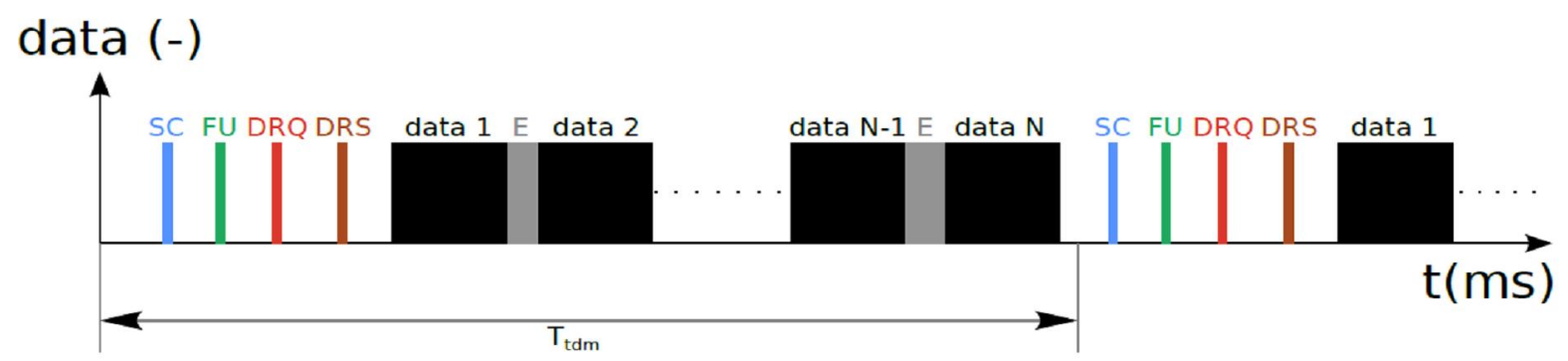

Fig. 10 - Time multiplexer.

The period of time multiplex $T_{\mathrm{tdm}}$ defines the time after which the same module starts with transmission of new measured data. This period should be so exactly determined to enable to transfer all measured data into the control unit and to execute the exchange of synchronization messages according the PTP protocol. To increase a possibility of another data collision, the "error window" $\mathrm{E}$ is implemented.

The control unit of the designed and developed distributed measuring system is a PC, which provides a simple unified interface for its handling using the Ethernet. An example of a window of the user interface is presented in Fig. 11.

\begin{tabular}{|c|c|c|c|c|c|}
\hline \multirow{2}{*}{\multicolumn{2}{|c|}{ Data Collector v3 }} & & & & \\
\hline & \multicolumn{5}{|c|}{ Device } \\
\hline Device List & \multicolumn{2}{|c|}{ Circular measuremen } & & & \\
\hline \# & Start & Stop & State & Channel & Received \\
\hline 1 & Start & Stop & running & $\mathrm{CHI}$ & $240 \mathrm{kB}$ \\
\hline 2 & Start & Stop & running & $\mathrm{CH} 2$ & $240 \mathrm{kB}$ \\
\hline 3 & Start & Stop & running & $\mathrm{CH} 3$ & $240 \mathrm{kB}$ \\
\hline 4 & Start & Stop & running & $\mathrm{CH}_{6}$ & $4 \mathrm{kB}$ \\
\hline 5 & Start & Stop & running & $\mathrm{CH} 7$ & $8 \mathrm{kB}$ \\
\hline 6 & Start & Stop & stopped & $\mathrm{CHB}$ & $0 \mathrm{kB}$ \\
\hline 7 & Start & Stop & running & $\mathrm{CH} 15$ & $12 \mathrm{kB}$ \\
\hline 8 & Start & Stop & running & $\mathrm{CHI} 6$ & $12 \mathrm{kB}$ \\
\hline
\end{tabular}

Fig. 11 - An example of user interface.

\section{CONCLUSION}

The executed research proved that a precise synchronisation can be reached in industrial distributed systems even without special and expensive network devices. The fluctuation of time stamps in order of microseconds was achieved using the optimal implementation of the IEEE 1588 Precise Time Protocol. The possibility of application of GPS for synchronization in large system was also successfully tested. A low-cost GPS receiver LEA-6T uBlox was used for this purpose.

The packet delay measuring device (the PTP tester) was designed and developed to enable to test the real packet delay in a network, where PTP protocol is implemented. Two versions were realized - the first one for small networks using direct synchronization and the second one for large networks using GPS synchronization. The both versions were successfully tested. The accuracy of the packet delay measurement was better than $40 \mathrm{~ns}$. The PTP tester was fruitfully utilised for packet delay measurement of network components as well as for measurement in large networks.

The gained experience was applied in design of a prototype of a low cost distributed system with precise synchronization. In this case, the delay fluctuation was decrease to several microseconds using a modified way for transmission of the synchronization messages, which is based on a time multiplex.

\section{ACKNOWLEDGMENT}

The research described in this paper has been financed by the TAČR (Technology Agency of the Czech Republic) under the Grant No. TA01010988, Timing synchronous distributed systems for data acquisition and process control. 


\section{REFERENCES}

[1] M. A. Lombardi, L. M. Nelson, A. N. Novick and V. S. Zhang, Time and frequency measurements using the global positioning system, Cal Lab: The International Journal of Metrology, (8) 3 (2001), pp. 26-33.

[2] IEEE Std 1588-2008, IEEE Standard for a Precision Clock Synchronization Protocol for Networked Measurement and Control Systems, The Institute of Electrical and Electronics Engineers, Inc., New York, 2008.

[3] L. Cosart, Precision packet delay measurements using IEEE 1588v2, in Proceedings of the IEEE International Symposium on Precision Clock Synchronization for Measurement, Control and Communication, (ISPCS'2007), 2007, pp. 85-91.

[4] L. Cosart, Packet network timing measurement and analysis using an IEEE 1588 probe and new metrics, in Proceedings of the IEEE International Symposium on Precision Clock Synchronization for Measurement, Control and Communication, (ISPCS'2009), 2009, pp. 1-6.

[5] Texas Instruments, DP83630 Precision PHYTER - IEEE 1588 Precision Time Protocol Transceiver, Texas Instruments, Tech. Rep., 2011, Access mode: www.ti.com/lit/ds/ symlink/dp83630.pdf.

[6] D. W. Allan, Time and frequency (timedomain) characterization, estimation, and prediction of precision clocks and oscillators, IEEE Transaction on Ultrasonics, Ferroelectrics and Frequency Control, (UFFC34) 6 (1987), pp. 647-654.

[7] MTI-Milliren Technologies, 210 Series OCXO - Mini OCXO in a 14 Pin DIP Package, MTIMiliren Technologies, INC., Tech. Rep., 2012, Access mode: www.mti-milliren.com/ pdfs/210.pdf.

[8] J. Breuer, V. Vigner, J. Roztočil, Precise packet delay measurement in an Ethernet network, Measurement, (54) (2014), pp. 215-221.

[9] V. Vigner, J. Breuer, Precise synchronization in large distributed systems, in Proceedings of the IEEE $7^{\text {th }}$ International Conference on Intelligent Data Acquisition and Advanced Computing Systems. (IDAACS'2013), Berlin, Germany, 12-14 September 2013, pp. 226-230.

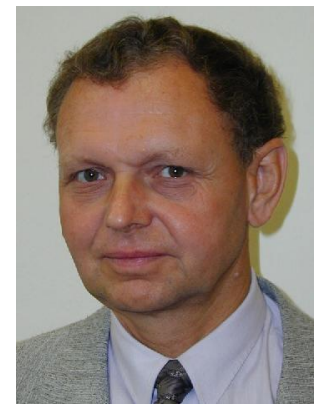

Vladimír Haasz, full professor at the Dept. of Measurement and at the University Centre for Energy Efficient Buildings of the Czech Technical University in Prague, member of the IMEKO TC-4 - Measurement of Electrical Quantities, member of the IEEE Instrumentation \& Measurement Society.

$\mathrm{He}$ is interested in measurement of dynamic parameters of $A D$ modules and in measuring systems.

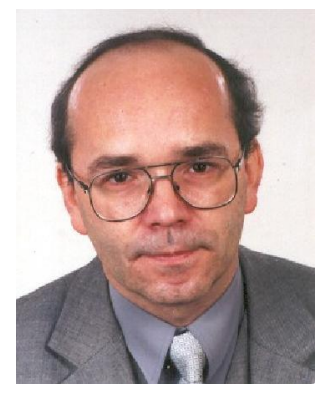

Jaroslav Roztočil received the Ph.D. degree in Measure-ment and Instrumentation from the Faculty of Electrical Engineering, Czech Technical University in Prague. Since 1981, he has been working at the Dept. of Measurement and was appointed as an Associate Professor in 1999.

$\mathrm{He}$ is interested in the metrology of time and frequency, the testing of the dynamic quality of $A D$ modules, the virtual measuring instrument design and in the development of measurement system software. He is a member of the International Union of Radio Science, a member of IEEE Instr. \& Meas. Society.

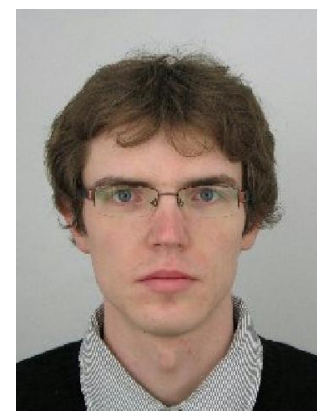

Jan Breuer graduated from the Faculty of Electrical Engineering Czech Technical University in Prague. Since 2009 he has been Ph.D. student at the Department of Measurement of CTU, FEE. $\mathrm{He}$ is interested in precise time synchronization in distributed systems.

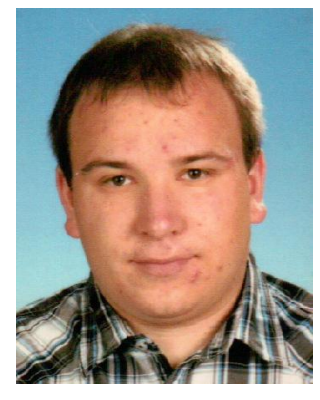

Vojtěch Vigner graduated from the Faculty of Electrical Engineering, Czech Technical University in Prague. Since 2010 he has been Ph.D. student at the Department of Measurement of CTU, FEE. His primary research interest deals with time and frequency stability and synchronization. 